\title{
Immunohistochemical analysis of microsomal glutathione S-transferase 1 and clusterin expression in lens epithelial cells of patients with pseudoexfoliation syndrome
}

\author{
JOANNA STAFIEJ $^{1}$, MARTA HAŁAS-WIŚNIEWSKA ${ }^{2}$, MAGDALENA IZDEBSKA ${ }^{2}$, \\ MACIEJ GAGAT ${ }^{2}$, DARIUSZ GRZANKA ${ }^{3}$, ALINA GRZANKA ${ }^{2}$ and GRAŻYNA MALUKIEWICZ ${ }^{1}$
}

\begin{abstract}
${ }^{1}$ Department of Ophthalmology, Faculty of Medicine, Nicolaus Copernicus University Collegium Medicum in Bydgoszcz, 85-094 Bydgoszcz; ${ }^{2}$ Department of Histology and Embryology, Faculty of Medicine, Nicolaus Copernicus University

Collegium Medicum in Bydgoszcz, 85-092 Bydgoszcz;

${ }^{3}$ Department of Dermatology, Sexually Transmitted Diseases and Immunodermatology, Faculty of Medicine, Nicolaus Copernicus University Collegium Medicum in Bydgoszcz, 85-094 Bydgoszcz, Poland
\end{abstract}

Received September 22, 2016; Accepted November 24, 2016

DOI: $10.3892 /$ etm.2017.4085

\begin{abstract}
Pseudoexfoliation syndrome (PEX) is an age-associated, sight disorder affecting elastic fibers in the eye and visceral organs but its exact etiology remains unknown. The purpose of the current study was to determine the morphology and ultrastructure of lens epithelial cells (LECs), and to use immunohistochemistry to examine localization of microsomal glutathione S-transferase 1 (MGST1) and clusterin. Anterior lens capsules were obtained from 24 patients (13 PEX and 11 controls) who underwent phacoemulsification. Immunohistochemistry was performed, using antibodies against MGST1 and clusterin, to determine their expression. The morphology and ultrastructure of LECs were evaluated by light and transmission electron microscopy, respectively. The PEX LECs were characterized by significantly lower MGST1 $(\mathrm{P}=0.0001)$ and clusterin expression $(\mathrm{P}=0.0005)$ compared with the control group patients. PEX LECs were also observed to have significantly increased thickness compared with the control group patients $(\mathrm{P}=0.0002)$. The current findings suggest that low MGST1 and clusterin expression levels may be an early clinical indicator of PEX, and that oxidative stress may serve an important role, but that the specific etiology of this disease has yet to be revealed.
\end{abstract}

Correspondence to: Dr Joanna Stafiej, Department of Ophthalmology, Faculty of Medicine, Nicolaus Copernicus University Collegium Medicum in Bydgoszcz, Marii Sklodowskiej-Curie 9, 85-094 Bydgoszcz, Poland

E-mail: joanna.stafiej@wp.pl

Key words: pseudoexfoliation syndrome, anterior lens capsules, microsomal glutathione S-transferase 1, clusterin, oxidative stress

\section{Introduction}

Pseudoexfoliation syndrome (PEX) was first described by Lindberg in 1917, but its exact etiology remains unknown (1). PEX is a common, age-associated systemic disorder of elastic fibers, affecting the lungs, liver, kidney, gall bladder and cerebral meninges $(2,3)$. Cardio- and cerebrovascular diseases, such as angina, aortic aneurysm and dementia have been linked to PEX (4-6); however, this association remains controversial (7-9).

The prevalence of pseudoexfoliation syndrome is known to vary widely between geographic regions; for instance, the Reykjavik Eye Study in Iceland reported an overall prevalence of $10.6 \%$ in patients over 50 years of age increasing to $40.6 \%$ in individuals over 80 years of age (10). Another population-based study, which was conducted in the Blue Mountains, west of Sydney, Australia, revealed an overall prevalence of 2.3\% in individuals over 49 years of age (11). Between these, the reported prevalence in Greece is $27 \%$ among age-associated cataract patients (12).

Pseudoexfoliation material has been identified in many human organs (2-6). Epidemiological data reveal that this disease occurs worldwide, and that prevalence increases with age; however, PEX is not an integral part of normal aging (13). PEX manifests in eyes through the accumulation of an abnormal fibrillar material on the extracellular matrix of tissues of the anterior segment. The pathological material appears as concentric rings of greyish fringes and flakes on the pupillary border of the iris and the peripheral lens capsule $(14,15)$. These deposits are diagnosed clinically via slit lamp examination, but it is unclear how this material is produced and accumulates.

Previous studies have reported an increased incidence of intraoperative complications during extracapsular cataract extraction in the eyes of patients with PEX compared with the eyes of patients without this syndrome (16-20). Serious complications in these patients appear to be predominantly caused by zonular weakness (21). Weakened zonules may manifest clinically as iridodonesis, phacodonesis, anterior chamber depth asymmetry or spontaneous lens subluxation 
or dislocation $(16,22,23)$. Intraoperatively, patients with PEX are at greater risk of zonular dialysis, posterior capsule tear, vitreous loss and displaced nucleus or fragment in the vitreous body; postoperatively, these patients have a higher incidence of inflammation in the form of increased aqueous flare and cellular response, fibrin reaction, posterior synechiae, posterior capsule opacification, anterior capsule phimosis and late intraocular lens decentration and dislocation (22). Phacoemulsification has become a typical procedure within routine cataract surgery, and previous studies have demonstrated an increased incidence of intraoperative, immediate postoperative and late complications caused by cataract surgery in the eyes of patients with PEX syndrome $(21,24,25)$. The pathology of PEX is therefore hypothesized to alter capsular rigidity, elasticity and other biomechanical properties of the eyes $(26,27)$.

One of the main secondary effects of PEX is blockage of the aqueous outflow mechanism of the eye (28). This may lead to elevation of intraocular pressure, causing loss of vision due to glaucomatous damage to the optic nerve, which is the second leading cause of irreversible blindness worldwide (29).

Although the causes of PEX are not well understood at the molecular level and the specific pathogenic mechanism(s) behind PEX are unknown, it is hypothesized that inflammation and oxidative stress are partially responsible $(30,31)$. The cellular antioxidant defense system comprises enzymatic antioxidants, including repair enzymes such as microsomal glutathione S-transferase 1 (MGST1). A role of clusterin in preventing the deposition of pseudoexfoliative material has also been proposed (32).

Therefore, the aims of the present study were to determine the morphology and ultrastructure of epithelial lens cells in PEX (using light and transmission electron microscopy, respectively) and to evaluate the expression of MGST1 and clusterin by immunohistochemistry (IHC).

\section{Materials and methods}

Tissue extraction. In the present study, anterior lens capsules and adherent lens epithelial cells (LECs) were obtained from 24 patients (comprising $13 \mathrm{PEX}$ and 11 control patients) who underwent routine cataract surgery over the course of 3 months between January and March 2015 in the Department of Ophthalmology, Nicolaus Copernicus University Collegium Medicum in Bydgoszcz (Poland). Written informed consent was obtained from each patient prior to tissue sample acquisition, and approval of the study was granted by the Bioethics Committee of the Nicolaus Copernicus University Collegium Medicum in Bydgoszcz. Two groups of patients were categorized, as follows: i) Patients with typical PEX [with deposits of pathological material observed, during slit lamp examination, as concentric rings of greyish fringes and flakes on the pupillary border of the iris and the peripheral lens capsule $(14,15)]$; ii) control patients with no clinical signs of this disease.

Following phacoemulsification, surgical specimens were immediately fixed in $2 \%(\mathrm{w} / \mathrm{v})$ paraformaldehyde (Serva Electrophoresis GmbH, Heidelberg, Germany), dehydrated in graded ethanol concentrations (Avantor Performance Materials, Gliwice, Poland) and embedded in paraffin. Tissue was then cut into thin sections (5-nm) on a Reichert microtome (Reichert Technologies, Depew, NY, USA), placed on
SuperFrost Plus microscopic slides and used in IHC staining (Thermo Fisher Scientific, Inc., Waltham, MA, USA).

IHC staining. Surgical specimens of human anterior lens capsules with adherent LECs on the above slides were immersed in xylene, xylene/ethanol and series of ethanol in descending concentrations (100-50\%; Avantor Performance Materials, Gliwice, Poland). For antigen retrieval, tissue on slides was placed in Target Retrieval Solution (Dako; Agilent Technologies, Inc., Santa Clara, CA, USA) and irradiated in a microwave at $800 \mathrm{~W}$ for $10 \mathrm{~min}$ and $400 \mathrm{~W}$ for $10 \mathrm{~min}$. The EnVision System-HRP (AEC+), used in accordance with the manufacturer's protocol, was applied for IHC staining (cat. no. K4008; Dako; Agilent Technologies, Inc.). Non-specific binding between the primary antibodies and the tissue was prevented using Peroxidase Blocking Reagent (Dako; Agilent Technologies, Inc.) at room temperature for $5 \mathrm{~min}$. The samples were exposed to antibodies and incubated in a humid chamber at room temperature for $30 \mathrm{~min}$. IHC was performed using rabbit antibodies against MGST1 (dilution, 1:1,000; cat. no. HPA044840; Sigma-Aldrich, Merck Millipore, Darmstadt, Germany) and clusterin (dilution, 1;1,000; cat. no. SAB3500199; Sigma-Aldrich; Merck Millipore). Following a series of washes, the slides were incubated with horseradish peroxidase-labelled polymer anti-rabbit for $1 \mathrm{~h}$ at room temperature (cat. no. K4008; Dako, Agilent Technologies, Inc.). The visualization of bound antibodies was performed using AEC+ substrate chromogen at room temperature for $15 \mathrm{~min}$ (as above). Counterstaining was performed using Mayer's hematoxyline $(10 \mathrm{~min}$, room temperature; Dako; Agilent Technologies, Inc.) and the slides were mounted using Aqua-Poly/Mount (Polysciences, Inc., Warminster, PA, USA). The surgical specimens were examined using an Eclipse E800 microscope (Nikon Corporation, Tokyo, Japan) with NIS-Elements Viewer v. 3.30 image analysis system and a CCD camera (DS-5Mc-U1; Nikon Corporation).

Transmission electron microscopy. To observe the morphological changes in the anterior lens capsule and LECs on the ultrastructural level, the surgical specimens were fixed in $2.5 \%$ glutaraldehyde (30 min, room temperature; Polysciences, Inc., Warminster, PA, USA), washed three times with $0.1 \mathrm{M}$ sodium cacodylate buffer (Carl Roth $\mathrm{GmbH}$ and Co. KG, Karlsruhe, Germany) and post-fixed with $\mathrm{OsO}_{4}(1 \%, 1 \mathrm{~h}$; Serva Electrophoresis $\mathrm{GmbH}$, Heidelberg, Germany) in the same buffer at room temperature. Next, the tissues were dehydrated in a graded series of ethanol (30-90\%; Avantor Performance Materials) and acetone (90-100\%; Avantor Performance Materials). Following this, the material was embedded in epoxy resin (glycidether 100; Carl Roth $\mathrm{GmbH}$ and Co. KG) with the addition of accelerator [2,4,6-tris (dimethylaminomethyl) phenol-30; Carl Roth GmbH and Co. KG] and hardeners (methyl nadic anhydride and DDSA; Carl Roth $\mathrm{GmbH}$ and Co. $\mathrm{KG}$ ), polymerized for $24 \mathrm{~h}$ at $37^{\circ} \mathrm{C}$, then for $120 \mathrm{~h}$ at $65^{\circ} \mathrm{C}$. Finally, the material was cut into ultra-thin sections using a Reichert Om U3 ultramicrotome (Leica Microsystems, Inc., Buffalo Grove, IL, USA) and placed on copper grids (Sigma-Aldrich; Merck Millipore). These ultra-thin slices were stained in uranyl acetate (Ted Pella, Inc., Redding, CA, USA) and analyzed using a transmission electron microscope (JEM-100 CXII; JEOL, Ltd., Tokyo, Japan). 
Thickness of cells and quantitation of IHC staining. Changes in the thickness of the LECs were analyzed on slides from human anterior lens capsules with adherent LECs, both from patients with PEX and from patients without clinical signs of these disease. To determine the mean thickness of the LECs, 100 micrographs were evaluated in each group; a measurement was recorded of the straight-line distance between the basolateral and the apical domains of cells, through the center of the cell nucleus. This analysis was performed using Fiji software (ImageJ 1.47i; imagej.nih.gov/ij/).

The IHC expression intensity of MGST1 and clusterin was also measured using Fiji software with a Colour Deconvolution plug-in, on 100 micrographs obtained with the same CCD camera parameters. The IHC images were distributed to individual color channels by vectors specific to AEC staining, and the value of the integrated density was defined as the intensity of the IHC reaction multiplied by the area of positive signal for the appropriate protein. The results were averaged for control and PEX cases.

Statistical analysis. The data are presented as the mean \pm standard error of the mean. Statistical evaluation of significant differences between the groups of patients was performed using a two-tailed Mann-Whitney U test (for the length of cells and the intensity of AEC IHC staining). P<0.05 was considered to indicate a statistically significant difference, and GraphPad Prism 5.0 (GraphPad Software, Inc., La Jolla, CA, USA) was used for these statistical analyses.

\section{Results}

Staining and quantitation of IHC. The IHC staining of MGST1 and clusterin in paraffin-embedded surgical specimens were first determined by light microscopy (Figs. 1 and 2, respectively). Positive MGST1 expression was visible in adherent LECs in control patients (Fig. 1A and B). However, examination of IHC labeling of this protein in PEX patients revealed an absence or only traces of IHC labeling (Fig. 1C and D, respectively). Notably, in the same paraffin-embedded anterior lens capsules and adherent LECs, the accumulation of pseudoexfoliation material was detected (Fig. 1D). MGST1 expression was significantly lower in PEX patients compared with control group patients $(\mathrm{P}<0.001)$. The PEX LECs were characterized by a lower level of MGST1 expression compared with the control (Fig. 3A).

The LECs in the control group also revealed higher expression of clusterin, whereas the PEX patients demonstrated absent or weakly positive clusterin-staining (Fig. 2C and D, respectively). Two types of clusterin immunoreactivity are presented, in both disease and control cases, in order to demonstrate the variation in staining intensity; the light microscopic observations were supported by a statistical analysis of the IHC reaction intensity of clusterin. As reported in Fig. 3B, the intensity of clusterin staining was statistically higher in the LECs of patients compared with the control group $(\mathrm{P}<0.001$; Fig. 3B).

IHC quantitation illustrated that the immunoreactivity of MGST1, as determined by the protein expression level, was similar in a few control group cases as in patients with PEX; for this reason, the disease history of selected patients was investigated (Fig. 4). The collected data revealed that a number

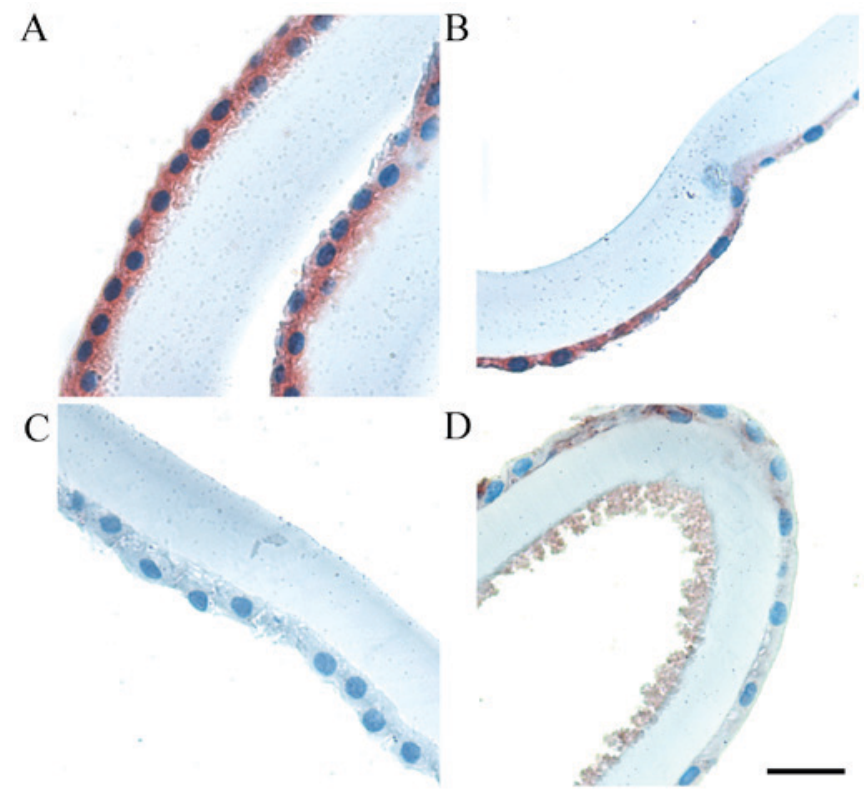

Figure 1. Immunohistochemical staining of MGST1 in (A, B) control patients without clinical symptoms of PEX; and patients with PEX with (C) an absence or (D) trace labeling of MGST1. Bar, $20 \mu \mathrm{m}$. PEX, pseudoexfoliation syndrome; MGST1, microsomal glutathione S-transferase 1.
A

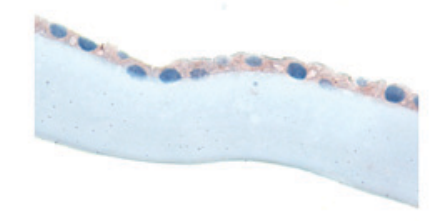

C

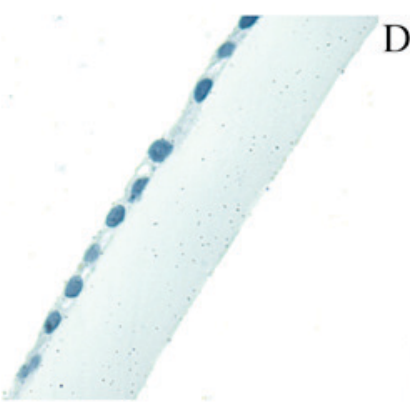

B

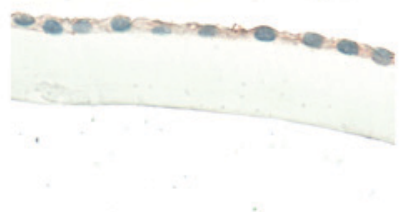

D

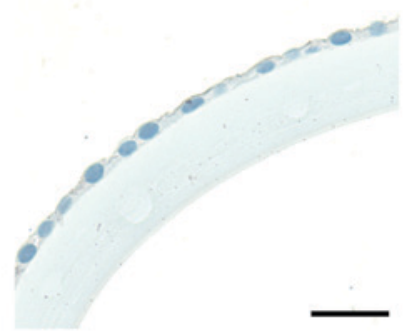

Figure 2. Immunohistochemical staining of clusterin in (A and B) control patients without clinical symptoms of PEX; and patients with PEX with (C) an absence or (D) trace labeling of clusterin. Bar, $20 \mu \mathrm{m}$. PEX, pseudoexfoliation syndrome.

of patients from control groups were classified as patients diagnosed as PEX after 2 years. Furthermore, two patients classified as control patients have since been diagnosed with glaucoma. These data are reported in Fig. 4.

Thickness of cells. As the mechanism of accumulation of pseudoexfoliation material, and in light of the present evaluation of IHC images and differences in the LECs, the differences in the thickness of cells were investigated. PEX LECs were characterized by a statistically significant increase in the vertical 


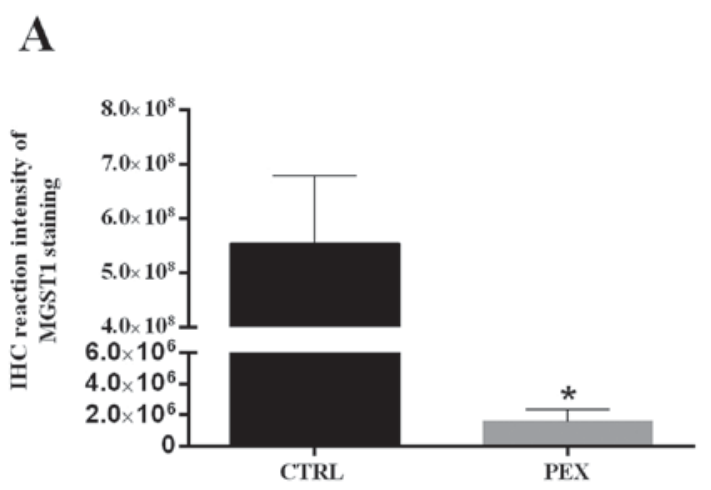

B

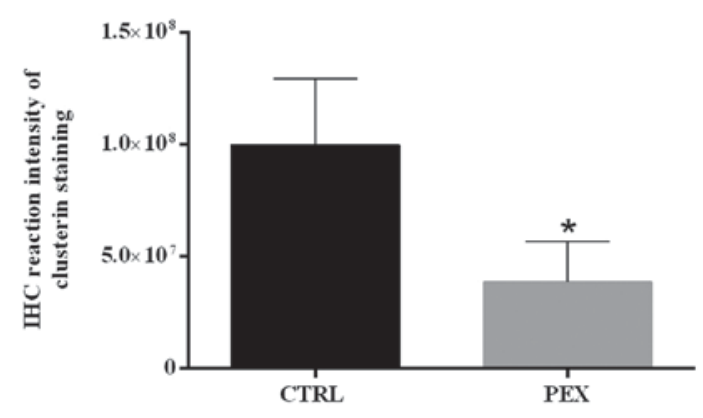

Figure 3. Statistical analysis of IHC reaction intensity of (A) MGST1 and (B) clusterin, expressed as mean \pm standard error of the mean. ${ }^{*} \mathrm{P}<0.05$ vs. control cells; Mann-Whitney U test. IHC, immunohistochemical; CTRL, control; PEX, pseudoexfoliation syndrome; MGST1, microsomal glutathione S-transferase 1.

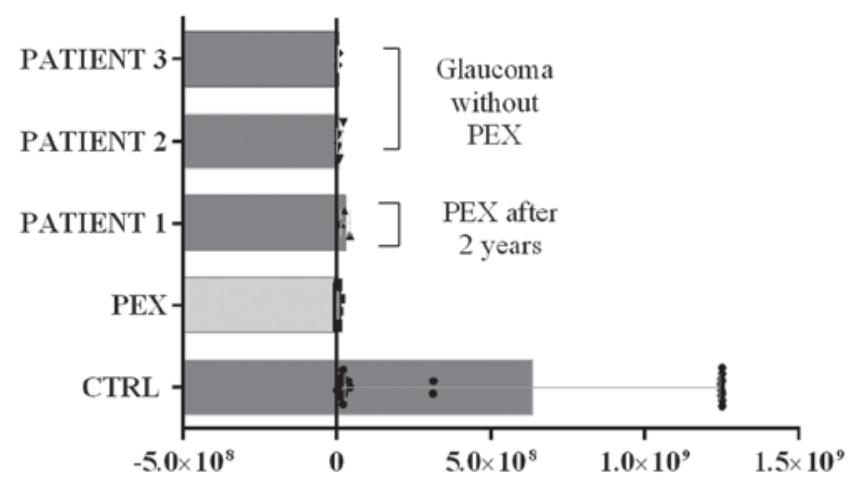

Relative IHC reaction intensity of MGST1 staining

Figure 4. Statistical analysis of IHC reaction intensity of MGST1 and in selected cases, expressed as mean \pm standard error of the mean. ${ }^{*} \mathrm{P}<0.05$ vs. control cells; Mann-Whitney U test. IHC, immunohistochemical; CTRL, control; PEX, pseudoexfoliation syndrome; MGST1, microsomal glutathione S-transferase 1.

thickness compared with control cells ( $\mathrm{P}<0.001$; Fig. 5), which may suggest upregulated secretion and/or changes in organization of junctions between LECs.

Transmission electron microscopy. In order to confirm the differences in the thickness of the cells, increased secretion and changes in the organization of cell-cell junctions, transmission electron microscopy was used. The above results were

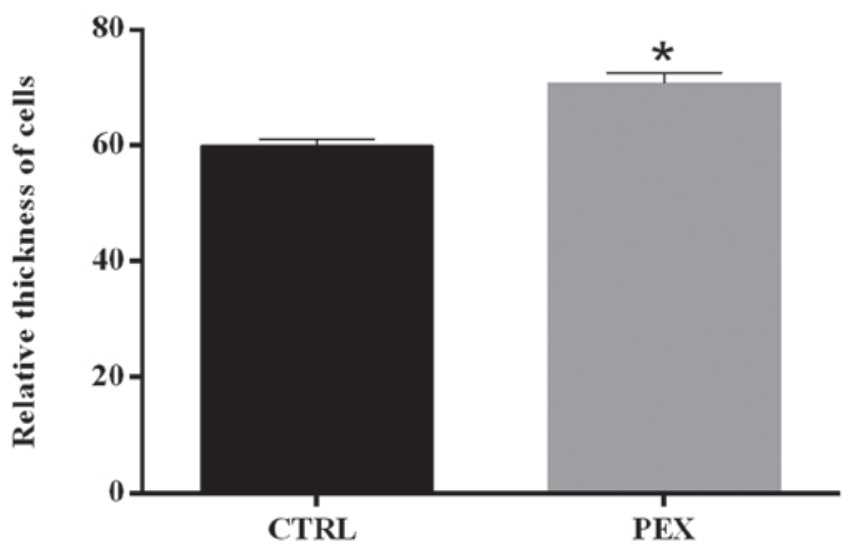

Figure 5. Thickness of cells, expressed as mean \pm standard error of the mean. ${ }^{*} \mathrm{P}<0.05$ vs. control cells; Mann-Whitney U test. CTRL, control; PEX, pseudoexfoliation syndrome.

confirmed, and are illustrated in Fig. 6. The normal lenses of control group patients are presented in Fig. 6A. The evaluation of morphological alterations in LECs revealed that control LECs and their nuclei were characterized by a flattened and stretched appearance (Fig. 6B and C). Accumulation of PEX material was observed in the front side of the lens (Fig. 6D) and PEX LECs appeared to be thicker and contained oval nuclei (Fig. 6E and F). Furthermore, ultrastructural analysis of PEX LECs revealed a markedly increased number of electron-dense secretory granules and enlargement of the cell-cell junctional area (Fig. 6E and F).

\section{Discussion}

Several mechanisms of pseudoexfoliation material formation are possible, but a molecular background is more frequently suggested (30,33-35). Oxidative stress is believed to contribute to the pathogenesis of many diseases. Reactive oxygen species (ROS) are formed as normal metabolic products and are important in normal cellular functioning, but their production may be increased under pathological conditions and cause cellular damage $(3,36)$.

Exogenous factors (e.g., exposure to environmental chemicals, radiation, and atmospheric oxygen) may increase their production. The imbalance between ROS production and the antioxidant defense mechanisms results in oxidative stress leading to cellular damage $(37,38)$. Lobo et al (39) and Sies (40) distinguished three levels of the antioxidant defense system, which is comprised of various enzymes such as superoxide dismutase, catalase and glutathione peroxidase, the low molecular weight antioxidants such as vitamins, and enzymes involved in this pathway such as aldehyde dehydrogenase 1 and repair enzymes such as glutathione peroxidase and microsomal glutathione S-transferase 1 .

The present results demonstrated a significantly lower MGST1 IHC reaction intensity in the PEX group compared with the control group ( $\mathrm{P}=0.0001)$. Strzalka-Mrozik et al (41) revealed significantly higher mRNA levels of SOD2, ALDH1A1, and MGST1 in the anterior lens capsules of patients with PEX and cataracts compared with control cataracts subjects. Zenkel et al (42) reported that the expression 


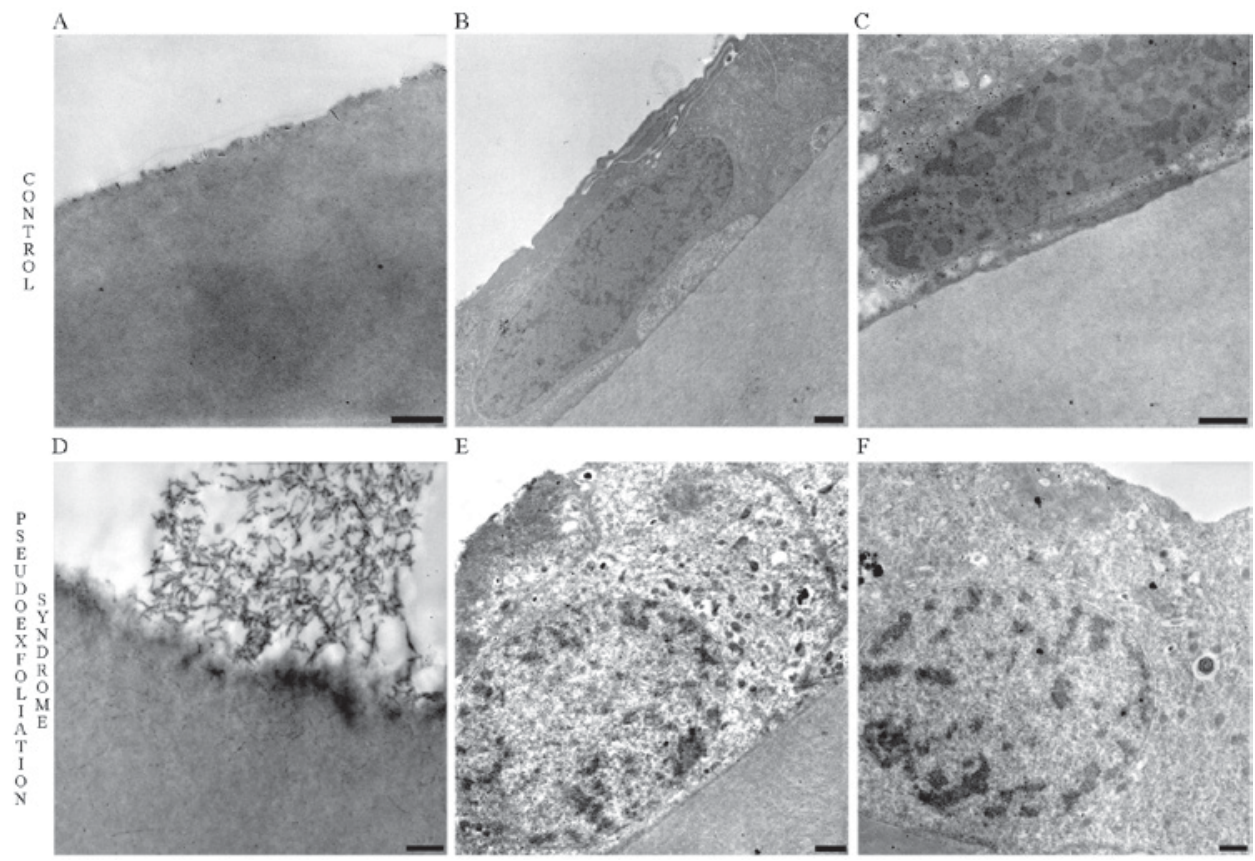

Figure 6. Transmission electron microscopy of the (A) lens capsule and (B and C) the lens epithelial cells in control patients. (D) The lens capsule indicating accumulation of pseudoexfoliative material and (E and F) the lens epithelial cells in PEX patients. Bar, $50 \mu \mathrm{m}$. PEX, pseudoexfoliation syndrome.

of MAPKp38, heat shock proteins (HSP40, HSP60) and superoxide dismutase (SOD2) were increased up to threefold in PEX specimens. In contrast, a large set of cytoprotective gene products, including antioxidant defense enzymes (the glutathione S-transferases MGST1 and GSTT1), ubiquitin-conjugating enzymes (UBE2A, UBE2B), the DNA repair protein MLH1 and the stress-inducible transcription factor GADD153, were found to be consistently downregulated up to threefold in PEX specimens on both the mRNA and protein levels (42). These findings correspond with the present results.

The available data suggest that chronic oxidative stress, in combination with weakened cytoprotective and repair strategies, affects the abnormal matrix metabolism by induction of a persistent proinflammatory state and activation of the profibrotic growth factor TGF-beta1. Oxidative stress, therefore, appears to represent a modifiable risk factor in the management of patients with PEX syndrome/glaucoma (43).

In the current research, divergence in the distribution of IHC measurements and the immunoreactivity of MGST1 were observed in three cases from the control group. These were similar to the protein expression level in PEX patients and, therefore, their disease history was followed. Notably, one control patient developed PEX two years following study commencement and, in two others, glaucoma has been diagnosed in a longer term follow up. This suggests that it may be possible to detect pseudoexfoliation syndrome/glaucoma prior to its clinical manifestation.

Clusterin is a highly efficient extracellular chaperone, and its deficiency in the LECs of eyes with PEX may therefore promote the stress-induced aggregation and stable deposition of the characteristic pathologic extracellular matrix product (32). Zenkel et al (44) observed that clusterin levels in the aqueous humor were significantly reduced in the eyes of PEX patients compared with patients with normal and glaucomatous control eyes. The present findings confirm this observation. A marked immunopositive reaction for clusterin was observed in the control group, whereas the PEX patients were characterized by unstained or weakly positive LECs. The obtained relative results were statistically significant $(\mathrm{P}=0.0005)$.

In fact, subtle chronic inflammatory processes, also termed 'molecular inflammation', have been suggested to underlie the causes of numerous age-associated chronic degenerative diseases, such as Alzheimer's disease, atherosclerosis and cardiovascular disorders (45). In accordance with this hypothesis, one major causative factor in chronic tissue injury is believed to be oxidative stress; in older individuals, oxidative stress combined with weakened cytoprotective strategies and stress-response mechanisms may lead to a persistent proinflammatory state (30). One of the multiple functions of clusterin is to act as an inhibitor of the complement system, an important mediator of inflammation (46). Significant clusterin deficiency in the LECs of patients with PEX compared with the LECs of patients without PEX may suggest an impaired cytoprotective mechanism or its depletion in pseudoexfoliation syndrome.

The mechanisms controlling capsular synthesis and the specification of the basal surface of lens cells are unclear. During lens reconstitution from epithelium/capsule fragments in the developing chick, cells that lose contact with the basement membrane undergo anoikis (apoptosis due to loss of matrix attachment) while cells attached to the capsule fragment migrate to reestablish their normal polarity within the eye (47). It is known that the elasticity within the capsule is directly correlated with its thickness (48). All cells involved in the exfoliation syndrome process demonstrated common ultrastructural signs of active fibrillogenesis and metabolic activation, such as increased vesicular transport to the cell surface, extracellular material formation within invaginations of cellular surfaces and a prominent rough endoplasmic 
reticulum (49). To our knowledge, this is the first report comparing LEC thickness and nuclei shape in patients with PEX to controls. Significant differences in cell morphology alterations may be due to the high metabolic activation of LECs in PEX, but this requires additional investigation.

The present findings suggest that low MGST1 and clusterin expression level may be an early clinical sign of pseudoexfoliation syndrome and that oxidative stress may serve an important role in the etiology of PEX, but additional investigation may serve to provide further clarity.

\section{References}

1. Lindberg JG: Clinical investigations on depigmentation of the pupillary border and translucency of the iris: In cases of senile cataract and in normal eyes in elderly persons. Acta Ophthalmol Suppl 190: 1-96, 1989.

2. Schlötzer-Schrehardt UM, Koca MR, Naumann GO and Volkholz H: Pseudoexfoliation syndrome. Ocular manifestation of a systemic disorder? Arch Ophthalmol 110: 1752-1756, 1992.

3. Streeten BW, Li ZY, Wallace RN, Eagle RC Jr and Keshgegian AA: Pseudoexfoliative fibrillopathy in visceral organs of a patient with pseudoexfoliation syndrome. Arch Ophthalmol 110: $1757-1762,1992$.

4. Mitchell P, Wang JJ and Smith W: Association of pseudoexfoliation syndrome with increased vascular risk. Am J Ophthalmol 124: 685-687, 1997.

5. Schumacher S, Schlötzer-Schrehardt U, Martus P, Lang W and Naumann GO: Pseudoexfoliation syndrome and aneurysms of the abdominal aorta. Lancet 357: 359-360, 2001.

6. Ritland JS, Egge K, Lydersen S, Juul R and Semb SO: Exfoliative glaucoma and primary open-angle glaucoma: Associations with death causes and comorbidity. Acta Ophthalmol Scand 82: 401-404, 2004

7. Allingham RR, Loftsdottir M, Gottfredsdottir MS, Thorgeirsson E, Jonasson F, Sverisson T, Hodge WG, Damji KF and Stefánsson E: Pseudoexfoliation syndrome in Icelandic families. Br J Ophthalmol 85: 702-707, 2001.

8. Stafiej J, Malukiewicz G, Lesiewska-Junk H, Rość D and Kaźmierczak K: Endothelial cell markers in patients with pseudoexfoliation syndrome. ScientificWorldJournal 2012: 863949, 2012.

9. Tarkkanen A: Is exfoliation syndrome a sign of systemic vascular disease? Acta Ophthalmol 86: 832-836, 2008.

10. Arnarsson A, Damji KF, Sverrisson T, Sasaki H and Jonasson F Pseudoexfoliation in the Reykjavik Eye Study: Prevalence and related ophthalmological variables. Acta Ophthalmol Scand 85: 822-827, 2007.

11. Mitchell P, Wang JJ and Hourihan F: The relationship between glaucoma and pseudoexfoliation: The Blue Mountains Eye Study. Arch Ophthalmol 117: 1319-1324, 1999.

12. Andrikopoulos GK, Mela EK, Georgakopoulos CD, Papadopoulos GE, Damelou AN, Alexopoulos DK and Gartaganis SP: Pseudoexfoliation syndrome prevalence in Greek patients with cataract and its association to glaucoma and coronary artery disease. Eye(Lond) 23: 442-447, 2009.

13. Forsius H: Exfoliation syndrome in various ethnic populations. Acta Ophthalmol Suppl 184: 71-85, 1988.

14. Ritch R: Exfoliation syndrome and occludable angles: Trans Am Ophthalmol Soc 92: 848-855, 1994.

15. Damji KF, Bains HS, Stafansson E, Loftsdottir M, Sverrisson $\mathrm{T}$, Thorgeirsson E, Jonasson $\mathrm{F}$, Gottfredsdottir $\mathrm{M}$ and Allingham RR: Is pseudoexfoliation syndrome inherited? A review of genetic and nongenetic factors and a new obserwation. Ophthalmic Genet 19: 175-185, 1998.

16. Alfaiate M, Leite E, Mira J and Cunha-Vaz JG: Prevalance and surgical complications of pseudoexfoliation syndrome in Portuguese patients with senile cataract. J Cataract Refract Surg 22: 972-976, 1996.

17. Guzek JP, Holm M, Cotter JB, Cameron JA, Rademaker WJ, Wissinger DH, Tonjum AM and Sleeper LA: Risk factors for intraoperative complicatons in 1000 extracapsular cataract cases. Ophthalmology 94: 461-466, 1987.

18. Abbasoğlu OE, Hoşal B, Tekeli O and Gürsel E: Risk factors for vitreous loss in cataract surgery. Eur J Ophthalmol 10: 227-232, 2000 .
19. Drolsum L, Haaskjold E and Davanger M: Pseudoexfoliation syndrome and extracapsular cataract extraction. Acta Ophthalmol (Copenh) 71: 765-770, 1993.

20. Lumme $P$ and Laatikainen L: Exfoliation syndrome and cataract extraction. Am J Ophthalmol 116: 51-55, 1993.

21. Hyams M, Mathalone N, Herskovitz M, Hod Y, Israeli D and Geyer O: Intraoperative complications of phacoemulsification in eyes with and without pseudoexfoliation. J Cataract Refract Surg 31: 1002-1005, 2005

22. Küchle M, Viestenz A, Martus P, Händel A, Jünemann A and Naumann GO: Anterior chamber depth and complications during cataract surgery in eyes with pseudoexfoliation syndrome. Am J Ophthalmol 129: 281-285, 2000.

23. Freissler K, Küchle M and Naumann GO: Spontaneous dislocation of the lens in pseudoexfoliation syndrome. Arch Ophthalmol 113: 1095-1096, 1995.

24. Sufi AR, Singh T, Mufti AA and Rather MH: Outcome of Phacoemulsification in patients with and without Pseudoexfoliation syndrome in Kashmir. BMC Ophthalmol 12: 13, 2012.

25. Ascaso FJ, Huerva V and Grzybowski A: Epidemiology, etiology, and prevention of late IOL-capsular bag complex dislocation: Review of the literature. J Ophthalmol 2015: 805706, 2015.

26. Guo S, Gewirtz M, Thaker R and Reed M: Characterizing pseudoexfoliation syndrome through the use of ultrasound biomicroscopy. J Cataract Refract Surg 32: 614-617, 2006.

27. Ünsal E, Eltutar K, Muftuoglu I, Akcetin TA and Acar Y: Ultrasound biomicroscopy in patients with unilateral pseudoexfoliation. Int J Ophthalmol 8: 754-758, 2015.

28. Johnson M: 'What controls aqueous humour outflow resistance'? Exp Eye Res 82: 545-557, 2006.

29. Barbosa AM, Frare AB, Costa NB, Silva RE and Moura KK: GSTM1 polymorphism in patients with primary open-angle glaucoma. Genet Mol Res 11: 3256-3262, 2012.

30. Zenkel M, Lewczuk P, Jünemann A, Kruse FE, Naumann GO and Schlötzer-Schrehardt U: Proinflammatory cytokines are involved in the initiation of the abnormal matrix process in pseudoexfoliation syndrome/glaucoma. Am J Pathol 176: 2868-2879, 2010.

31. Tanito M, Kaidzu S, Takai Y and Ohira A: Status of Systemic oxidative stresses in patients with primary open-angle glaucoma and pseudoexfoliation syndrome. PLoS One 7: e49680, 2012.

32. Burdon KP, Sharma S, Hewitt AW, McMellon AE, Wang JJ, Mackey DA, Mitchell P and Craig JE: Genetic analysis of the clusterin gene in pseudoexfoliation syndrome. Mol Vis 14: $1727-1736,2008$

33. Schlötzer-Schrehardt U: Molecular pathology of pseudoexfoliation syndrome/glaucoma-new insights from LOXL1 gene associations. Exp Eye Res 88: 776-785, 2009.

34. Elhawy E, Kamthan G, Dong CQ and Danias J: Pseudoexfoliation syndrome, a systemic disorder with ocular manifestations. Hum Genomics 6: 22, 2012.

35. Schlötzer-Schrehardt U: Genetics and Genomics of Pseudoexfoliation Syndrome/Glaucoma. Middle East Afr J Ophthalmol 18: 30-36, 2011.

36. Schlötzer-Schrehardt UM, Koca MR, Naumann GO and Volkholz H: Pseudoexfoliation syndrome. Ocular manifestation of a systemic disorder? Arch Ophthalmol 110: 1752-1756, 1992.

37. Comhair SA and Erzurum SC: Redox control of asthma: Molecular mechanisms and therapeutic opportunities. Antioxid Redox Signal 12: 93-124, 2010

38. Yildirim Z, Ucgun NI and Yildirim F: The role of oxidative stress and antioxidants in the pathogenesis of age-related macular degeneration. Clinics (Sao Paulo) 66: 743-746, 2011.

39. Lobo V, Patil A, Phatak A and Chandra N: Free radicals, antioxidants and functional foods: Impact on human health. Pharmacogn Rev 4: 118-126, 2010.

40. Sies H: Total antioxidant capacity: Appraisal of a concept. J Nutr 137: 1493-1495, 2007.

41. Strzalka-Mrozik B, Prudlo L, Kimsa MW, Kimsa MC, Kapral M, Nita M and Mazurek U: Quantitative analysis of SOD2, ALDH1A1 and MGST1 messenger ribonucleic acid in anterior lens epithelium of patients with pseudoexfoliation syndrome. Mol Vis 19: 1341-1349, 2013.

42. Zenkel M, Kruse FE, Naumann GO and Schlötzer-Schrehardt U: Impaired cytoprotective mechanisms in eyes with pseudoexfoliation syndrome/glaucoma. Invest Ophthalmol Vis Sci 48: 5558-5566, 2007.

43. Schlötzer-Schrehardt U: Oxidative stress and pseudoexfoliation glaucoma. Klin Monbl Augenheilkd 227: 108-113, 2010. 
44. Zenkel M, Kruse FE, Jünemann AG, Naumann GO and Schlötzer-Schrehardt U: Clusterin deficiency in eyes with pseudoexfoliation syndrome may be implicated in the aggregation and deposition of pseudoexfoliative material. Invest Ophthalmol Vis Sci 47: 1982-1990, 2006.

45. Maggio M, Guralnik JM, Longo DL and Ferrucci L: Interleukin-6 in aging and chronic disease: A magnificent pathway. J Gerontol Biol Sci Med Sci 61: 575-584, 2006.

46. Calero M, Rostagno A, Frangione B and Ghiso J: Clusterin and Alzheimer's disease. Subcell Biochem 38: 273-298, 2005.
47. Danysh BP and Duncan MK: The Lens Capsule. Exp Eye Res 88: 151-164, 2009.

48. Krag S and Andreassen TT: Mechanical properties of the human lens capsule. Prog Retin Eye Res 22: 749-767, 2003.

49. Zenkel M and Schlötzer-Schrehardt U: The composition of exfoliation material and the cells involved in its production. J Glaucoma 23 (8 Suppl 1): S12-S14, 2014. 